\title{
Noninvasive Imaging Study of Tracheobronchial Compression Caused By Tortuosity and Dilation of the Thoracic Artery
}

Rong-Zhen OuYang ${ }^{1}$, Mao Sheng ${ }^{2}$, Yu-Min Zhong ${ }^{1 *}$, Ai -Min Sun ${ }^{1}$, Qian Wang ${ }^{1}$, Wei Gao ${ }^{3}$, Hai-Sheng Qiu ${ }^{1}$ and Ming Zhu ${ }^{1}$

${ }^{1}$ Diagnostic Imaging Center, P.R. China

${ }^{2}$ Department of Radiology, Suzhou Children's hospital affiliated to Suzhou University, School of Medicine, No.303 Jing De Road, Suzhou, 215001, P.R. China

${ }^{3}$ Department of Pediatric Cardiology, Shanghai Children's Medical Center affiliated to Shanghai Jiao Tong University, School of Medicine, No.1678 Dong Fang Road, Shanghai 200127, P.R. China

\section{Abstract}

Objective: To demonstrate tracheobronchial compression caused by tortuous and dilated large and medium-sized thoracic arteries and the characteristics of these arteries by noninvasive imaging modalities.

Patients and Methods: Imaging and clinical data of six patients with tortuous, dilated large and medium-sized thoracic arteries (July 2007 to December 2013) were reviewed. The imaging data were mainly acquired by cardiac multi-slice computed tomography (MSCT) or/and cardiac magnetic resonance imaging (MRI). The number and location of tracheobronchial stenosis were noted, and the severity of stenosis was categorized. The characteristics of large and medium-sized thoracic arteries were also noted. Further, the correlation between the severity of tracheobronchial stenosis and tortuous, dilated large and medium-sized thoracic arteries was analyzed. One patient who was suspected to have arterial tortuosity syndrome underwent genetic analysis.

Results: All six patients demonstrated varying degrees of tortuosity and dilation of large and medium-sized thoracic arteries. Five were noted to have varying degrees of tracheobronchial compression and respiratory symptoms. Two have varying degrees of connective tissue abnormalities.

Conclusion: Tracheobronchial compression of tortuosity and dilation of large and medium-sized thoracic vascular origin is an uncommon and frequently unrecognized cause of respiratory distress in children. Noninvasive imaging modalities like MSCT and MRI could clearly demonstrate tracheobronchial compression and vascular abnormalities simultaneously. Attention should be given to tracheobronchial compression when disease management decisions are made, especially for determining surgical strategies and for those patients who are 6-9 months old.B.

Conclusions: MAB is frequent in patients with COPD and is associated with hypoxemia independent of other cardiovascular risk factors.

Keywords: Tracheobronchial compression; Tortuosity and dilation; Thoracic arteries; Connective tissue abnormalities

\section{Introduction}

Cardiovascular structures and airway anatomy are closely related; thus, tracheobronchial compression often occurs in some cardiovascular abnormalities, such as vascular rings, left pulmonary artery sling, and absent pulmonary valve, and could lead to recurrent respiratory infection, shortness of breath and wheezing [1-4]. It is founded that tracheobronchial compression can also be caused by certain rare vascular anomalies, such as tortuosity, dilation and aneurysm formation in large and medium-sized thoracic arteries, which are found in some rare connective tissue disorders such as arterial tortuosity syndrome (ATS), Loeys-Dietz syndrome (LDS) and Marfan syndrome (MFS) [511]. However, these disorders are frequently unfound or unrecognized in children especially when the patient was asymptomatic. Few previous reports have analyzed the relationship between airway findings and the underlying rare vascular anomalies. The condition of airways and vessels can provide valuable information needed for decision making during management of diseases, especially for preoperation decision making. Among the imaging means including US, radiography, bronchoscopy and angiography, computed tomography (CT) and magnetic resonance imaging (MRI) are the most powerful diagnostic tools for demonstrating tracheobronchial compression and vascular abnormalities simultaneously. The aim of this study is to demonstrate the tracheobronchial compression caused by the rare dilated and tortuous thoracic arteries together with the typical vascular characteristics in rare disorders which may be connective tissue disorders by using noninvasive imaging modalities--CT and MRI.

\section{Materials and Methods}

\section{Patients}

Our institutional review board approved of this retrospective research, which was conducted in compliance with the Health Insurance Portability and Accountability Act (HIPAA). The imaging and clinical data of six patients obtained between July 2007 and December 2013 demonstrated dilation and tortuosity of large and medium-sized thoracic arteries. The patients were three boys and three girls with age ranging from 4 months to 11 years (median age, 4 years). The imaging data were mainly acquired using cardiac multi-slice computed tomography (MSCT) and/or magnetic resonance imaging (MRI).

\section{MSCT imaging}

MSCT (Light Speed 16; GE Medical Systems, Milwaukee, WI) was used in two patients, one of whom underwent MSCT for follow-up

*Corresponding author: Yu-Min Zhong, Diagnostic Imaging Center, No 1678 Dong Fang Road, Shanghai Children's Medical Center, Shanghai, 200127, P.R. China, Tel: 8621-38626161-5001; Fax: 8621-58393915; E-mail: zyumin2002@yahoo.com

Received July 02, 2015; Accepted July 16, 2015; Published July 20, 2015

Citation: OuYang RZ, Sheng M, Zhong YM, Sun AM, Wang Q, et al. (2015) Noninvasive Imaging Study of Tracheobronchial Compression Caused By Tortuosity and Dilation of the Thoracic Artery. J Pulm Respir Med 5: 281. doi:10.4172/2161105X.1000281

Copyright: @ 2015 OuYang RZ, et al. This is an open-access article distributed under the terms of the Creative Commons Attribution License, which permits unrestricted use, distribution, and reproduction in any medium, provided the original author and source are credited. 
study after he underwent cardiac MRI one year ago. For the patients below 4 years, oral chloraldurate and/or intramuscular phenobarbital was used for sedation during scanning, and the scan was performed with free breathing. In patients aged above 4 years who could cooperate, the scan was performed under inspiration. The raw data were obtained with the following parameters: voltage, $120 \mathrm{kV}$; current, 80-120 mA; collimation, $0.625 \mathrm{~mm}$; table speed, $5.62 \mathrm{~mm} / \mathrm{s}$; rotation speed, $0.5 \mathrm{~s} /$ rotation; reconstruction interval, $0.3 \mathrm{~mm}$. Non-ECG triggering was performed using a nonionic contrast agent $(2 \mathrm{ml} / \mathrm{kg}, 400 \mathrm{mg} \mathrm{I} / \mathrm{ml})$ (iopamidol or iohexol) injected through the peripheral vein. When an arm vein was used, MSCT was performed in the caudo-cranial direction to decrease contrast medium-related artifacts and to achieve homogeneous contrast enhancement. When a foot vein was used, MSCT was performed in the cranio-caudal direction. The injection rate using a power injector was $0.8-2.5 \mathrm{ml} / \mathrm{s}$, according to the scan range. The scan delay was $13-18 \mathrm{~s}$ according to the site of injection and age. The MSCT raw data were processed on an off-line workstation (Advantage Windows 4.2; GE Healthcare, Milwaukee, WI) with a maximum intensity projection (MIP) algorithm and/or volume rendering (VR) and a minimum intensity projection (MinIP) algorithm.

\section{MRI imaging}

MRI was performed in five patients with a 1.5-T MR unit (Signa, General Electric Medical Systems; Achieva TX, Philips Healthcare, Best, Netherlands). When the age of the patient was below 4 years, oral chloraldurate and/or intramuscular phenobarbital was used for sedation during scanning, and the scan was performed with free breathing. A phased-array coil was used, and contrast-enhanced MR angiography was performed using a three-dimensional (3D) fast spoiled gradient echo sequence in the coronal plane with the following parameters: field of view (FOV), 300-360 mm; repetition time, $2.8 \mathrm{~ms}$; echo time, $1.8 \mathrm{~ms}$; flip angle, $35^{\circ} \mathrm{C} ; 248 \times 254$ matrix; slice thickness, $2.6 \mathrm{~mm}$; bandwidth, $196 \mathrm{kHz}$. The imaging time for each series ranged from 10 to $15 \mathrm{~s}$. Two to three consecutive sets of images were acquired to visualize the great vessels. 3D turbo field echo (3D-TFE) sequencing was performed in the coronal plane to observe the tracheobronchial tree. The parameters were as follows: Time of Repetition, $5.6 \mathrm{~ms}$; Time of Echo, $1.71 \mathrm{~ms}$; flip angle, $30^{\circ} \mathrm{C}$; FOV, $300-400 \mathrm{~mm}$; matrix size, $300 \times 300$; number of slices, 30-40; slice thickness, $2.0 \mathrm{~mm}$. Dimeglumine gadopentetate (Magnevist) was used as the contrast medium at a dose of $0.2 \mathrm{mmol} / \mathrm{kg}$ $(0.4 \mathrm{ml} / \mathrm{kg})$ body weight. The contrast medium was injected by hand or a power injector. The MRI raw data were also processed on an off-line workstation (Advantage Windows 4.2; Philips Medical Systems, Best, The Netherlands) with an MIP algorithm and/or VR.

Other imaging studies included plain chest radiography $(n=6)$, echocardiography $(n=4)$, and pulmonary and cardiac cine-angiography $(n=1)$.

\section{Results}

\section{Patient characteristics}

The clinical data are summarized in Table 1. All six patients were born of non-consanguineous, healthy parents. Five patients had recurrent respiratory symptoms (e.g., recurrent wheezing, stridor, respiratory infections, or respiratory distress) to some degree, and another one was referred to our institution because of occasional cough and fever. Two patients had connective tissue abnormalities, including hyperlaxity of the skin, hernia and joint laxity. The characteristics of the connective tissue and the vascular abnormalities in one patient are shown in Figure 1.
One patient, a 4-month-old boy, who suffered severe respiratory distress, was suspected to have ATS and underwent genetic analysis, but the results did not indicate particular gene mutations of this syndrome. This boy suffered death two months later for sudden severe respiratory distress.

\section{Imaging findings}

The imaging data of the arteries are displayed in Table 2. Tortuosity and dilation of the ascending aorta, aortic arch, descending aorta and brachiocephalic artery were present to some degree in all six patients. Relative stenosis was found in the aortic arch in two patients and in the left pulmonary artery in one patient. The vascular lesion was demonstrated clearly on both MSCT and MRI scans (Figure 2).

The status of tracheobronchial compression is displayed in Table 3. Five patients had tracheobronchial compression to some degree, caused by tortuous and dilated large and medium-sized thoracic arteries (Figure $3)$. The severity of the tracheobronchial compression was categorized as mild $(<25 \%)$, moderate $(25-75 \%)$, or severe $(>75 \%)$ by comparing the area of stenosis with that of the adjacent normal trachea or bronchus using a lung window (window width, $1500 \mathrm{HU}$; window level, -500 HU) [12] on the axial section. Among these six patients, five (5/6) had mild compression located at the upper one third of trachea, one $(1 / 6)$ had severe compression located at the upper two thirds of trachea, three (3/6) had mild compression located at the lower one third of trachea and three (3/6) had mild left main bronchial compression. Radiography of the chest was performed in all six patients and revealed varying degrees of widening of the superior mediastinum. Echocardiography performed in four patients and revealed normal intracardiac anatomy and partial abnormal vascular manifestations (tortuosity, dilation and elongation of the large arteries).

\section{Discussion}

Respiratory complaints such as dyspnea and recurrent respiration are commonly seen in children with cardiac or vascular abnormalities. In children with persistent recurrent respiratory distress, unexplained atelectasis, or extubation failure, the possibility of airway compression should be strongly suspected [13-16]. In this study, the findings imply that the location of tracheobronchial compression caused by tortuous and dilated large and medium-sized thoracic arteries can be very different. It showed that tracheal especially upper tracheal compression was almost caused by lesions of ascending aorta, aortic arch, brachiocephalic artery and main pulmonary artery whereas obstruction of the left main bronchus was mainly caused by lesions of the left pulmonary artery, aorta. There were no cases of obstruction of the right main bronchus in this study, but it could be caused by dilated main pulmonary artery and right pulmonary artery. Although feeding difficulty was not found in this study either, it may be found in patients with co-existing esophageal compression while the large and mediumsized thoracic arteries dilated enough , as some studies have noted [15].

The clinical and imaging data in this study demonstrated that the non-invasive imaging methods both MSCT and MRI can clearly demonstrate the imaging features of large and medium-sized thoracic arteries with dilation, tortuosity and elongation and tracheobronchial compression simultaneously. Although MSCT remains an ionizing procedure and vascular studies require the use of intravenous contrast injection, the rapid examination time and light sedation it requires makes it a priority for patients with severe respiratory stress. Moreover, MSCT can clearly demonstrate lung lesions and tracheobronchial compression simultaneously.

MRI has a number of advantages for evaluating cardiovascular 
Citation: OuYang RZ, Sheng M, Zhong YM, Sun AM, Wang Q, et al. (2015) Noninvasive Imaging Study of Tracheobronchial Compression Caused By Tortuosity and Dilation of the Thoracic Artery. J Pulm Respir Med 5: 281. doi:10.4172/2161-105X.1000281

Page 3 of 5

\begin{tabular}{|c|c|c|c|c|c|}
\hline Patient & Sex & Age & Imaging modality & Chief complaints & Physical characteristics \\
\hline 1 & M & $4 \mathrm{M}$ & MSCT & Recurrent wheezing, respiratory infection & $\begin{array}{l}\text { Soft and hyperelastic skin and hydrocele of the tunica } \\
\text { vaginalis, down-slanting palpebral fissures, broad } \\
\text { forehead, hypertelorism, prominent upper lips }\end{array}$ \\
\hline 2 & M & $7 \mathrm{Y}$ & $\begin{array}{l}\text { MSCT } \\
\text { MRI }\end{array}$ & $\begin{array}{c}\text { Recurrent wheezing since } 6 \text { months with } \\
\text { cyanosis after normal activities, respiratory } \\
\text { tract infection }\end{array}$ & $\begin{array}{l}\text { L2-4 III/VI systolic murmur and wheezing at the end of } \\
\text { expiration, soft and hyperelastic skin }\end{array}$ \\
\hline 3 & $\mathrm{~F}$ & $4 \mathrm{Y}$ & MRI & $\begin{array}{l}\text { Recurrent wheezing and respiratory } \\
\text { infections }\end{array}$ & L2-4 III/VI systolic murmur \\
\hline 4 & $\mathrm{~F}$ & $10 Y$ & MRI & $\begin{array}{c}\text { Stridor before } 6 \text { years, recurrent respiratory } \\
\text { infection }\end{array}$ & L2-4 III/VI systolic murmur, broad forehead \\
\hline 5 & $\mathrm{~F}$ & $4 \mathrm{Y}$ & MRI & Occasional cough and fever & No obvious abnormalities \\
\hline 6 & $\mathrm{~F}$ & $11 \mathrm{Y}$ & MRI & Recurrent respiratory infection & No obvious abnormalities \\
\hline
\end{tabular}

MSCT: multi-slice computed tomography; MRI: magnetic resonance imaging

Table 1: Major clinical information and imaging modalities for the seven patients.
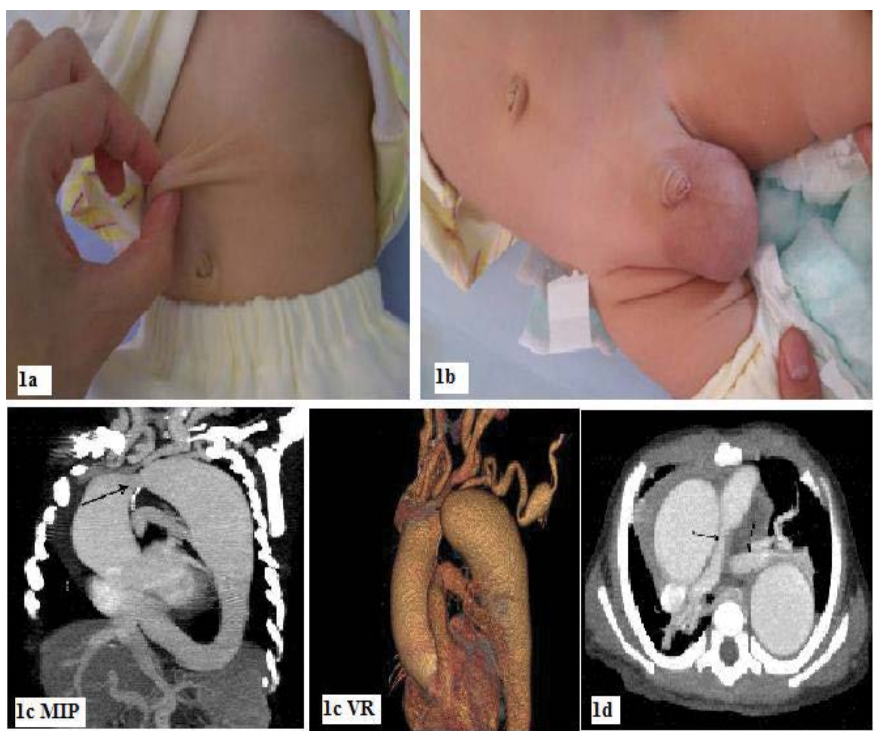

Figure 1: Findings for a 4-month-old boy with arterial dilation, tortuosity and elongation. (a) Physical examination showed soft and hyperelastic skin. (b) t also showed hydrocele of the tunica vaginalis. (c) Multi-slice computed tomography (MSCT) coronal maximum intensity projection (MIP) and volume reconstruction $\square$ VR demonstrated dilation and tortuosity of the ascending aorta, descending aorta, and brachiocephalic artery, and relative stenosis of the aortic arch (arrow). (d) MSCT axial MIP reconstruction demonstrated dysplasia of the pulmonary artery (arrow).

\begin{tabular}{|c|c|c|c|}
\hline & Tortuosity & Elongation & Dilation \\
\hline Ascending aorta & 3 & 3 & 4 \\
\hline Aortic arch & 6 & 5 & 3 \\
\hline Descending aorta & 5 & 5 & 4 \\
\hline Brachiocephalic artery & 3 & 3 & 3 \\
\hline Pulmonary artery & 0 & 0 & 0 \\
\hline
\end{tabular}

Table 2: Number and location of characteristic lesions of large and medium-sized thoracic arteries in MRI or MSCT scans.

abnormalities including the apparent fact that it does not use ionizing radiation [17] and its larger FOV can demonstrate multiple artery anomalies. In four of our patients, MRI clearly displayed the proximal abdominal aorta and the distal aortic arch branches and in one patient, the dilation and tortuosity of renal artery were clearly observed. It can also clearly demonstrate tracheobronchial compression as the MSCT does as $3 \mathrm{D}$ turbo field echo (3D-TFE) sequencing was performed in the coronal plane. However, it is not a good choice for the patients who have severe respiratory distress, who have heart pacemaker and who have metal implant in the scan field. In this study, most cases $(5 / 6)$ underwent MRI and the tracheas were clearly displayed.

Long-term follow-up of every three months was recommended for all six patients and cardiac MRI was the first recommendation for its advantage without ionization radiation. The respiratory symptoms including the degree and frequency of wheezing or stridor, respiratory infections and respiratory distress needed to be noted. Also, the lesion of both thoracic arteries and tracheobronchial compression requested to be paid close attention to. Bronchomalacia can occur from prolonged bronchial compression and tracheobronchial cartilage immaturity 
Citation: OuYang RZ, Sheng M, Zhong YM, Sun AM, Wang Q, et al. (2015) Noninvasive Imaging Study of Tracheobronchial Compression Caused By Tortuosity and Dilation of the Thoracic Artery. J Pulm Respir Med 5: 281. doi:10.4172/2161-105X.1000281
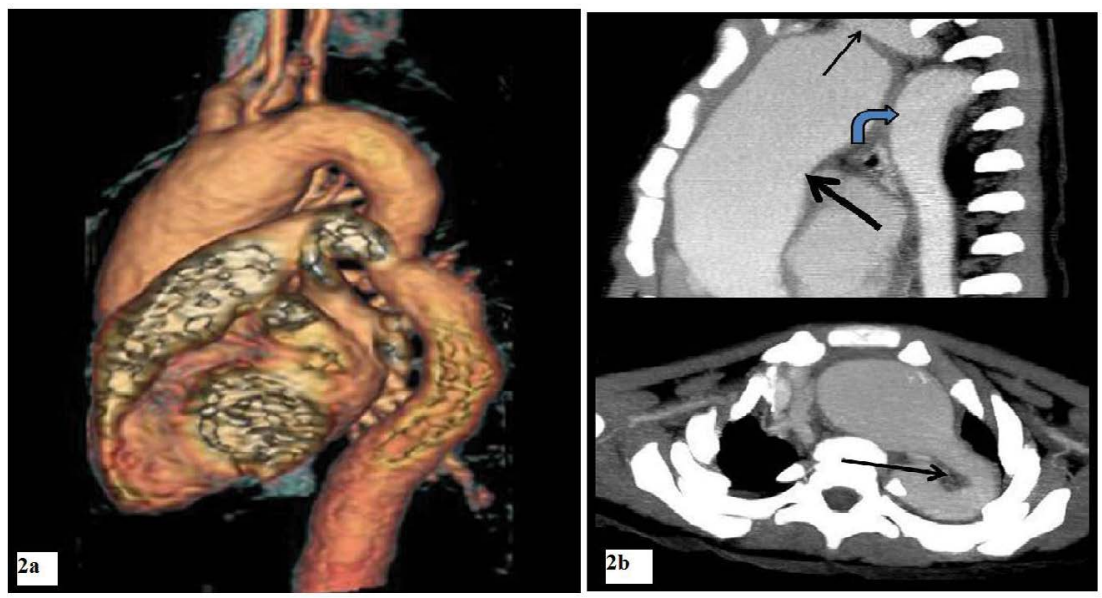

Figure 2: MSCT and MRI findings of abnormal large and medium-sized arteries. (a) Findings for a 4-year-old girl with arterial tortuosity elongation and dilation: contrast-enhanced magnetic resonance angiography (MRA) with volume rendering reconstruction demonstrates tortuosity and elongation of the ascending aorta and descending aorta (arrow). (b) Findings for a 17-month-old boy: Sagittal MSCT with MIP reconstruction (up) demonstrated dilation of the ascending aorta (wide arrow), local relative stenosis of the aortic arch (narrow arrow), and mild dilation of the descending aorta (bent arrow); axial MIP reconstruction (below)demonstrated tortuosity of the aortic arch (arrow).

\begin{tabular}{|c|c|c|c|c|}
\hline severity of stenosis & upper third of the trachea & middle third of the trachea & lower third of the trachea & Left main bronchus \\
\hline mild $(<\mathbf{2 5 \% )}$ & 5 & 0 & 3 \\
\hline moderate(25-75\%) & 0 & 0 & 0 \\
\hline severe $(>\mathbf{7 5} \%)$ & 1 & 1 & 0 \\
\hline
\end{tabular}

Five $(5 / 6)$ had mild compression located at the upper one third of trachea, one $(1 / 6)$ had severe compression located at the upper two thirds of trachea, three $(3 / 6)$ had mild compression located at the lower one third of trachea and three (3/6) had mild left main bronchial compression.

Table 3: Number and Severity of tracheobronchial compression in seven patients.
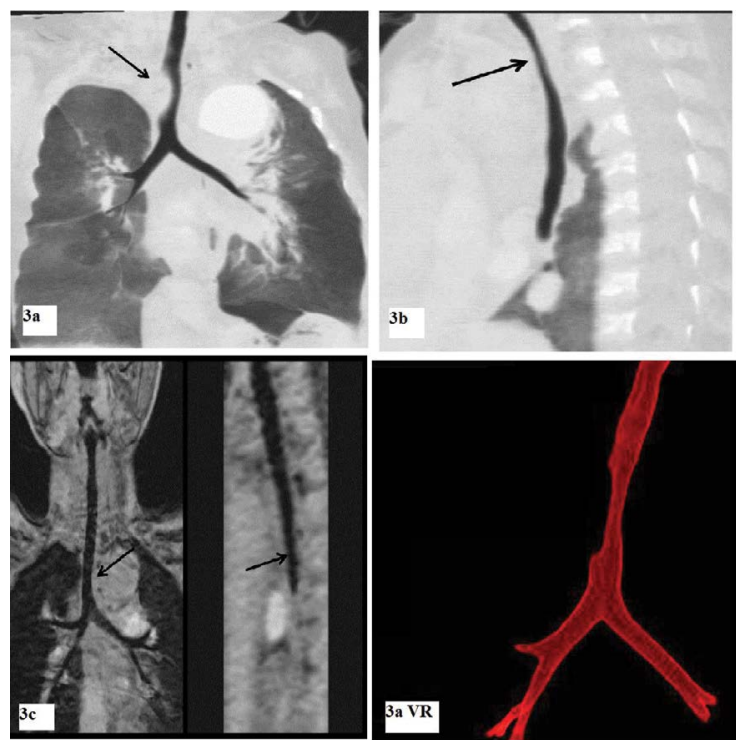

Figure 3: MSCT and MRI findings of the severity and location of tracheobronchial compression. (a) Findings for a 4-month-old boy with arterial tortuosity and dilation: Coronal MSCT with MinIP(3a MinIP) reconstruction and volume reconstruction $\square$ 3a VR $\square$ demonstrated moderate compression of the lower and middle thirds of the trachea. (b) Findings for a 7-year-old boy with arterial tortuosity and dilation: Sagittal MSCT with MinIP reconstruction demonstrated severe compression of the middle and upper thirds of the trachea. (c) Findings for a 10-year-old girl: Three-dimensional echo field (3D-TFE) magnetic resonance imaging (MR) with coronal and sagittal MinIP reconstruction demonstrated dilation of the ascending aorta and mild anterio-posterior compression of the lower third of the trachea (arrow). 
before the first 6-9 months of age, so, aggressive management as early as possible is needed for a favorable prognosis [4,18-20]. And cine and volume helical shuttle(VHS) computed tomography (CT), and spirometer-controlled cine magnetic resonance imaging(MRI ) are all good techniques for evaluation for tracheobronchomalacia [21] though it is a pity that in this study no special techniques for evaluation for tracheobronchomalacia, and we will evaluate tracheobronchomalacia for posterior and the follow up study.

Marked tortuosity, dilatation and aneurysms of the large and medium-sized arteries can be often found in some rare connective tissue disorders like ATS, LDS and MFS that present with similar typical vascular features and could cause tracheobronchial compression. In such cases, genetic analysis is necessary for differentiation [22].

\section{Conclusions}

Cardiovascular structures and airway anatomy are closely related; thus, tracheobronchial compression often occurs in some cardiovascular abnormalities, and could lead to recurrent respiratory infection, shortness of breath and wheezing [1-4]. Tracheobronchial compression can also be caused by certain rare vascular anomalies. Noninvasive imaging modalities such as MSCT and MRI with some special techniques can clearly demonstrate the anatomic features of arterial abnormalities and tracheobronchial compression, and the findings of airway should be considered when making decisions about disease management. Long-term follow-up is necessary for patients with arterial tortuosity and dilation because some syndromes may have life-threatening complications such as respiration distress caused by severe tracheobronchial stenosis or bronchomalacia, artery dissection and ruptured aneurysm. However, several connective tissue diseases such as ATS, LDS and MFS display similar clinical and imaging features, and genetic analysis is necessary to differentiate between these connective tissue diseases.

\section{References}

1. Zhong YM, Jaffe RB, Zhu M, Gao W, Sun AM, et al. (2010) CT assessment of tracheobronchial anomaly in left pulmonary artery sling. Pediatr Radiol 40: 1755-1762.

2. Berdon WE (2000) Rings, slings, and other things: vascular compression of the infant trachea updated from the midcentury to the millennium--the legacy of Robert E. Gross, MD, and Edward BD. Neuhauser, MD. Radiology 216: 624632.

3. Berdon WE, Baker DH (1972) Vascular anomalies and the infant lung: rings, slings, and other things. Semin Roentgenol 7: 39-64.

4. Zhong YM, Jaffe RB, Liu JF, Sun AM, Gao W, et al. (2014) Multi-slice computed tomography assessment of bronchial compression with absent pulmonary valve. Pediatr Radiol 44: 803-809.

5. Ekici F, Uçar T, Fitöz S, Atalay S, Tutar E (2011) Cardiovascular findings in a boy with arterial tortuosity syndrome: case report and review of the literature. Turk J Pediatr 53: 104-107.

6. Ritelli M, Drera B, Vicchio M, Puppini G, Biban P, et al. (2009) Arterial tortuosity syndrome in two Italian paediatric patients. Orphanet J Rare Dis 4: 20
7. Satish G, Nampoothiri S, Kappanayil M (2008) Images in cardiovascular medicine. Arterial tortuosity syndrome: phenotypic features and cardiovascular manifestations. Circulation 117: e477-478.

8. Ou P, Marijon E, Bonnet D (2006) Images in cardiology. Arterial tortuosity syndrome in a newborn. Heart 92: 7.

9. Naunheim MR, Walcott BP, Nahed BV, MacRae CA, Levinson JR, et al. (2011) Arterial tortuosity syndrome with multiple intracranial aneurysms: a case report. Arch Neurol 68: 369-371.

10. Hoop R, Steinmann B, Valsangiacomo Buechel ER (2006) Cardiovascular findings in arterial tortuosity syndrome. Eur Heart J 27: 2045.

11. Cohen IR, Lajtha A, Lambris JD, Letti RP (2014) Progress in Heritable Sof Connective Tissue Diseases, Advances in Experimental Medicine and Biology. USA: Jaroslava Halper.

12. Quint LE, Whyte RI, Kazerooni EA, Martinez FJ, Cascade PN, et al. (1995) Stenosis of the central airways: evaluation by using helical CT with multiplanar reconstructions. Radiology 194: 871-877.

13. Lee SL, Cheung YF, Leung MP, Ng YK, Tsoi NS (2002) Airway obstruction in children with congenital heart disease: assessment by flexible bronchoscopy. Pediatr Pulmonol 34: 304-311.

14. Cerda J, Chacón J, Reichhard C, Bertrand P, Holmgren NL, et al. (2007) Flexible fiberoptic bronchoscopy in children with heart diseases: a twelve years experience. Pediatr Pulmonol 42: 319-324.

15. Turner A, Gavel G, Coutts J (2005) Vascular rings--presentation, investigation and outcome. Eur J Pediatr 164: 266-270.

16. Guillemaud JP, El-Hakim H, Richards S, Chauhan N (2007) Airway pathologic abnormalities in symptomatic children with congenital cardiac and vascular disease. Arch Otolaryngol Head Neck Surg 133: 672-676.

17. Krishnamurthy R (2009) The role of MRI and CT in congenital heart disease Pediatr Radiol 39 Suppl 2: S196-204.

18. Morris SA, Orbach DB, Geva T, Singh MN, Gauvreau K, et al. (2011) Increased Vertebral Artery Tortuosity Index Is Associated With Adverse Outcomes in Children and Young Adults With Connective Tissue Disorders. Circulation 124 388-396.

19. Hraska V, Photiadis J, Schindler E, Sinzobahamvya N, Fink C, et al. (2009) A novel approach to the repair of tetralogy of Fallot with absent pulmonary valve and the reduction of airway compression by the pulmonary artery. Semin Thorac Cardiovasc Surg Pediatr Card Surg Annu.

20. Berlinger NT, Lucas RV Jr, Foker J (1984) Pulmonary arteriopexy to relieve tracheobronchial compression by dilated pulmonary arteries. Ann Otol Rhino Laryngol 93: 473-476.

21. Ciet $P$, Wielopolski $P$, Manniesing $R$, Lever $S$, de Bruijne $M$, et al. (2014) Spirometer-controlled cine magnetic resonance imaging used to diagnose tracheobronchomalacia in paediatric patients. Eur Respir J 43: 115-124.

22. Loup O, Daubeney PE, Saggar A, Rubens M, Naqvi N, et al. (2013) Severe arterial tortuosity in an asymptomatic infant with coarctation. Circ Cardiovasc Imaging 6: 487-490. 